\title{
Angioscopic Observation of an Endoluminal Stent Graft: CT Imaging versus Angioscopic Imaging
}

\author{
Yasuhiko Kobayashi1 ${ }^{*}$, Takahiro Yamaguchi' ${ }^{2}$, Sei Komatsu ${ }^{3}$, Tomoki Ohara4, \\ Junichi Yoshida ${ }^{3}$, Mitsuhiko Takewa ${ }^{3}$, Satoru Takahashi ${ }^{3}$, Chikao Yutani ${ }^{5}$, \\ Kazuhisa Kodama ${ }^{3}$, Shizuo Yoshida ${ }^{6}$ \\ ${ }^{1}$ Departments of Cardiovascular Surgery, Amagasaki Central Hospital, Hyogo, Japan \\ ${ }^{2}$ Departments of Cardiovascular Surgery, Kawachi General Hospital, Osaka, Japan \\ ${ }^{3}$ Departments of Cardiology, Amagasaki Central Hospital, Hyogo, Japan \\ ${ }^{4}$ Ohara Internal Medicine and Cardiology Clinic, Hyogo, Japan \\ ${ }^{5}$ Departments of Pathology, Amagasaki Central Hospital, Hyogo, Japan \\ ${ }^{6}$ Departments of Surgery, Amagasaki Central Hospital, Hyogo, Japan \\ Email: "bw100kg@hotmail.co.jp
}

Received 2 March 2016; accepted 27 June 2016; published 30 June 2016

Copyright (C) 2016 by authors and Scientific Research Publishing Inc.

This work is licensed under the Creative Commons Attribution International License (CC BY).

http://creativecommons.org/licenses/by/4.0/

c) (i) Open Access

\begin{abstract}
We could observe the endoluminal stent graft (SG) following thoracic endovascular aneurysm repair (TEVAR) by a coronary artery angioscope and establish intravascular visualization of SG. The patient was a 70-year-old woman with the distal aortic arch aneurysm and the descending aortic aneurysm, and debranching TEVAR were performed. After 12 months follow up, urgent hospitalization was required for chest pain, and cardiac catheter examination with a coronary artery angioscope was performed. The endoluminal SG was observed. The observation in angioscope which is a video image is better than CT that is a still image, and observation in blood vessel or SG is possible. It may be possible to observe the endoluminal SG, allowing potential investigation of an endoleak, or the covering status of the SG with the native aortic vessel wall, or the state of intimal membrane formation in the endoluminal graft.
\end{abstract}

\section{Keywords}

Intravascular Visualization, Angioscopy, Endoluminal Stent Graft, Endovascular Aneurysm Repair

\footnotetext{
${ }^{*}$ Corresponding author.
}

How to cite this paper: Kobayashi, Y., Yamaguchi, T., Komatsu, S., Ohara, T., Yoshida, J., Takewa, M., Takahashi, S., Yutani, C., Kodama, K. and Yoshida, S. (2016) Angioscopic Observation of an Endoluminal Stent Graft: CT Imaging versus Angioscopic Imaging. World Journal of Cardiovascular Surgery, 6, 87-92. http://dx.doi.org/10.4236/wjcs.2016.66014 


\section{Introduction}

Angina and cardiac infarction were investigated by observing the coronary artery using a coronary artery angioscope [1] [2], and a new treatment concept [3]-[6] was proposed. Angioscopy is considered an excellent methodology for observing blood vessels, and the results are better than those seen with computed tomography (CT).

Observation of the aorta has been performed using an aortoscope, which is a modified fiberoptic endoscope [7], allowing development of new methodology to observe the clinical condition in aortic diseases. Additionally, examination and treatment of the stomach and gastrointestinal tract rely on examination of the great vessels using an angioscope.

In contrast, postoperative observation using static imagery following endovascular aneurysm repair is commonplace in a treatment protocol.

This study uses an angioscope and video monitoring to observe the endoluminal stent graft (SG) following thoracic endovascular aneurysm repair.

\section{Case Report}

The patient was a 70-year-old woman, referred to our hospital for general fatigue. According to the patient, when laboring for a number of consecutive days at work, an increasing weariness was experienced; a close inspection will have a near medicine, and was thus admitted to the hospital. Upon hospitalization, symptoms pertaining to the chest, back and breathing were not observed. Upon thoracoabdominal CT examination, a number of aneurysms, with maximum diameter of $46 \mathrm{~mm}$ in the distal aortic arch and $60 \mathrm{~mm}$ in the descending aorta Figure 1, were observed. The patient's physical state was stable, with symptoms of the chest and back not observed in the hospital. Further, the patient did not show symptoms of acute heart failure; however, significant coronary stenosis was observed in the left anterior descending (LAD) artery. Anaortic vessel expansion of 60 $\mathrm{mm}$ in the descending aortic artery was observed; however, no chest or heart symptoms were apparent. However, the patient was firm in requesting treatment to focus primarily on the aortic aneurysm. Following judgment that the coronary artery disease could be examined in passage, it was decided that TEVAR should be performed at the site of chronic aorta dissection (distal arch, descending-chest abdomen).

Firstly, axillo-axillary bypass (8 mm PTFE graft featuring external supporting rings) and debranching TEVAR $\left(G{ }^{\circledR}{ }^{\circledR}\right.$ TAG $\left.^{\circledR} 34 \mathrm{~mm}\right)$ were performed for distal arch aortic artery aneurysm on November 28, 2013. The next procedure, approximately two weeks later, was TEVAR performed for thoracoabdominal aortic aneurysm, with blood vessel prosthesis implantation performed for the aneurysm and the abdominal branching bypass (renal artery of both sides and the superior mesenteric artery). Post-operative outcomes were good for the most part, with no problems apparent upon CT, and noleak observed following the operation. The patient was discharged on December 28, 2013.

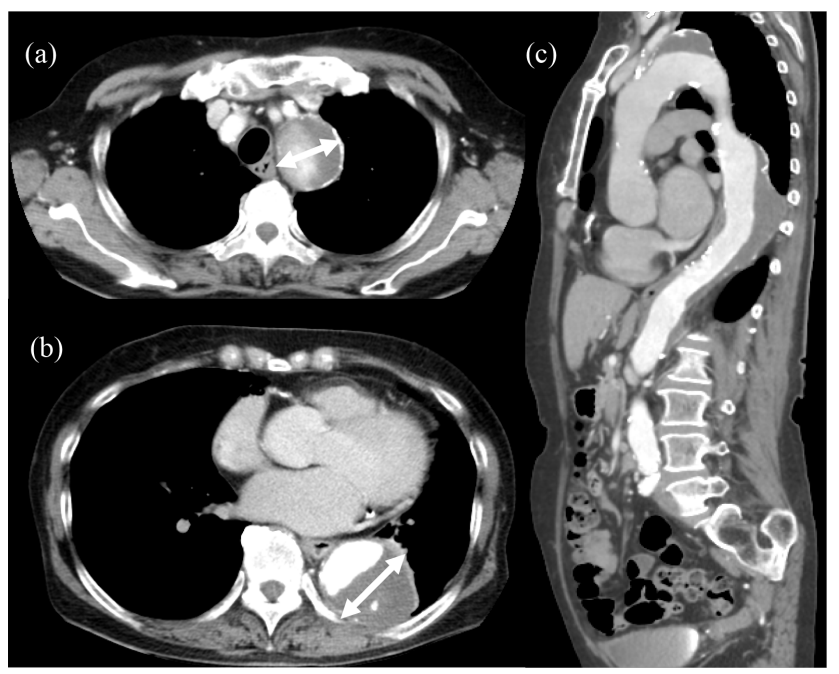

Figure 1. Preoperative thoracoabdominal CT image. (a) Maximum diameter of $46 \mathrm{~mm}$ in the distal aortic arch. (b) Maximum diameter of $60 \mathrm{~mm}$ in the descending aorta. (c) Sagittal plane. 
After 12 months follow up, urgent hospitalization was required on January 5, 2015 for chest pain. Cardiac catheter examination was performed, showing \#7: stenosis of 90\% - 99\%, \#1 and \#2 stenosis of 50\%, \#11 stenosis of 50\%; PCI was performed for stenotic coronary arteries. We decided to investigate the SG placed in the blood vessel, through use of a vascular angioscopy, following the catheter.

Vascular endoscopy was performed using a CCD camera endoscope with dual infusion system [8] with the thing of the non-obstructive type for use in the coronary arteries. When the intravascular observation was performed, a sizing catheter with marker was used as a method to identify the part of the blood vessel observed.

Firstly, the proximal element of the SG was observed. By observation of the central side of the aortic arch, the blood flow velocity is early; on the arch part lesser curvature side, the greater curvature side observed it, however, adaptation of the SG in the aorta was good. The internal aspect of the SG was observed using the vascular angioscopy, whilst drawing the angioscopy through the graft, in spite of being a spiral (Figure 2). Using the methodology developed with the coronary artery, it was possible to observe the endoluminal SG in the aortic artery, with no influence of the local blood velocity or infusion from the angioscopic camera tip, due to secure a field of vision. Thus, stent grafting may be observed, with four primary outcomes: 1) the endoluminal SG center side, 2) stent-stent graft junctions, 3) SG inside, and 4) distal SG sides. The bit of the SG was attached to the blood vessel lining membrane enough at the aortic arch part lesser curvature side and the major curvature side together.

Intimal hyperplasia was formed at the front and behind of the attached SG (Figure 3(a)).The "bird beak configuration [9] [10] is a radiological finding of a wedge shaped gap between the undersurface of the SG and the aortic wall occurred in the SG at the lesser curvature of the ascending aorta was absent and no leakage was observed in the SG. Further, intimal hyperplasia was observed to occupy the majority of endoluminal SG. The endoluminal SG was observed to contain substantial physiological debris, including frills, unevenness, thrombi, and hematomas (Figure 3(b)). Further, several places of the endoluminal SG did not appear to be coated with intimal hyperplasia (Figure 3(c)), and were exposed. The connection between the SG was coated with intimal hyperplasia. The distal element of the SG was also coated with intimal hyperplasia. A capsule tissue sample was taken by catheter aspiration, at several locations, on the endoluminal SG (Figure 4).

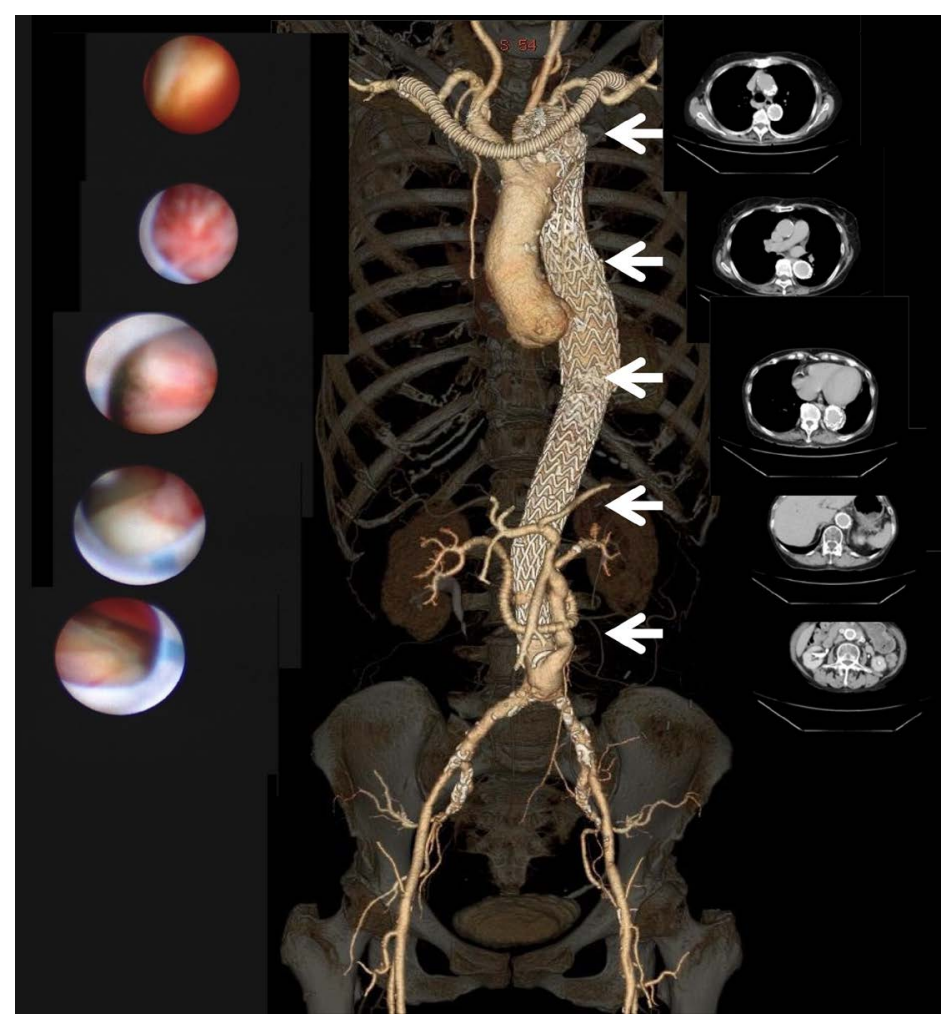

Figure 2. Postoperative 3-dimensional image showing the endoscopic findings at each site in the great vessels. 


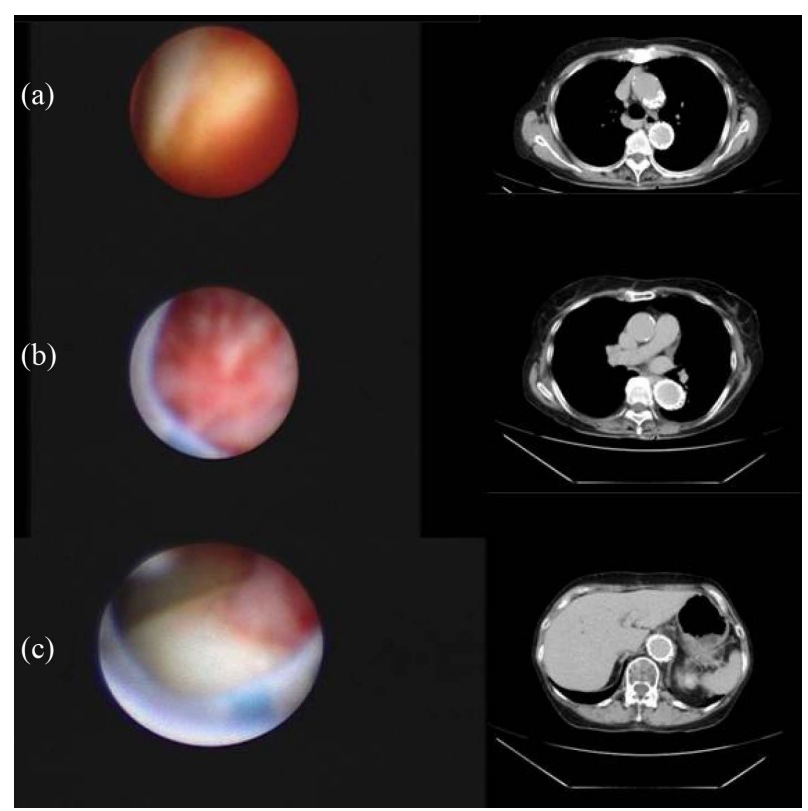

Figure 3. Comparison of the findings from CT and endoscopy at the same area in the SG. (a) Intimal hyperplasia can be observed, which was not identified on CT. (b) The SG contained substantial debris: frill, unevenness, thrombi, and hematoma. The gross findings of the inner surface of the SG are visible on CT. (c) Several elements of the internal surface of the SG were exposed, and not coated with intimal hyperplasia.

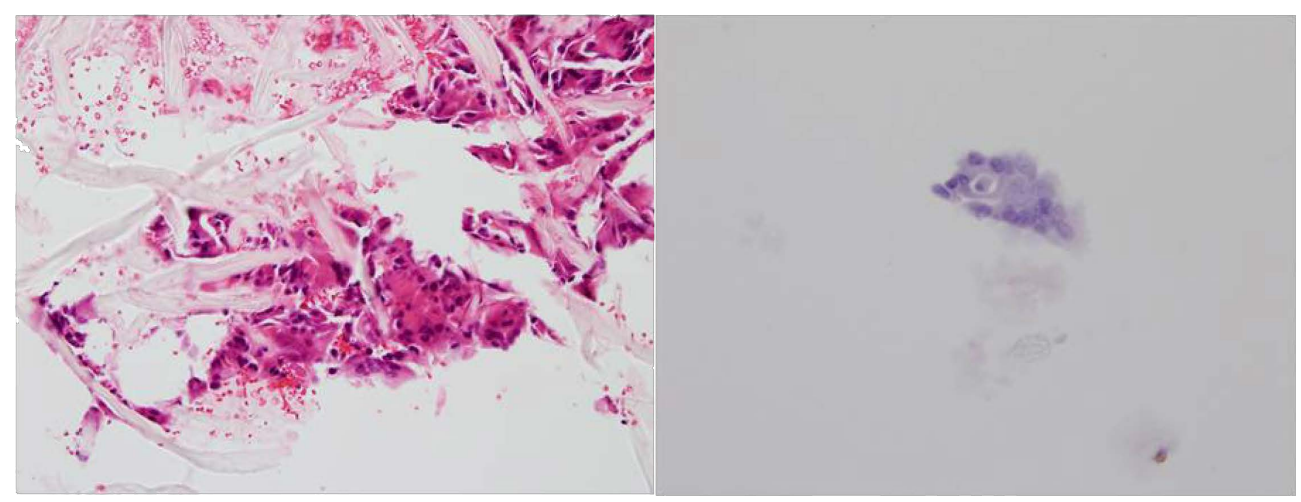

Figure 4. Hematoxylin-eosin stained section: A red blood cell was identified; however a number of cells appearing similar to macrophages were also observed.

After the PCI, there were no symptoms, and she was discharged from the hospital on the 12th day. The patient was followed up with clinical visits and follow-up CT. Postoperative CT showed no migration of the SG and there were no symptoms.

\section{Discussion}

Two types of coronary angioscopies exist; an obstructive type and non-obstructive type [11]. Previously, our institution has used non-obstructive angioscopy, in addition to a dual infusion system, as a means of obtaining more highly detailed imagery.

In this study, it was possible to observe the greater and lesser curvatures of the aorta, from the central aspect of the SG. Additionally, it was possible to observe the SG junction, the internal aspect of the SG, as well as the peripheral side of the SG. With vascular angioscopy it is possible to observe the following: 1) the endoluminal surface of the graft, allowing potential investigation of an endoleak, 2) the covering status of the SG with the native aortic vessel wall, 3) the state of intimal membrane formation in the endoluminal graft.

Endoleak [12] [13] remaining after TEVAR or abdominal endovascular aneurysm repair (EVAR), is a major 
clinical issue concern following endovascular repair procedures. There is also an animation check of magnetic resonance imaging, but the periodic check which put an interval period by the contrast CT is generally periodic inspection at intervals period. However, if observation is performed using a vascular angioscope, it may be possible to identify the cause of endoleak in the SG. Incomplete attachment of the SG can occur with ulceration, including ulcer-like projections (ULP) or calcifications on the native vessel wall, potentially resulting in an endoleak. In this case, we could observe long SG inside change by having detained a stent in the relatively long range.

Further, we were able to observe long endospore hyperplasia by having chosen a stent in the relatively long range. Previously, no such observation of the endoluminal surface of a long SG has been performed. This study examined the intimal membrane state of the aortic aorta, but intravascular beautiful cases were little. It was thought that state of the observing the intimal membrane of aortic aorta is a so-called arteriosclerosis pathological change. This state of the observing the intimal membrane of aortic aorta was dirty impression, and was including a different things such as the view a crack view, an unevenness view, a thrombus view, view like intramural hematoma, an internal membrane fluttering view or a blood outflow view from an internal membrane. It appears that hyperplasia of the internal membrane [14] is complete throughout the Gore-Tex endoluminal surface of the SG at a follow-up time of approximately one year. And this state was similar in the state in the aorta blood vessel of native and existed in the dirty state by the appearance. Potentially, the state of the native aorta may be a reflection of the restorative process occurring with the lining membrane hyperplasia. Poor progress of this restorative process may result in ulceration or ULP, leading to a potential for graft dissociation. In this study, we attempted to sample the plaque using catheter aspiration, with good results, suggesting that plaque sampling of the endoluminal surface of the SG is possible, and thus the potential for pathologic enquiry from the samples obtained (Figure 4). After TEVAR, we were able to observe stents placed in the great vessels using a vascular angioscope.

Hill et al. [7] reported that the first clinical use of the technique to visually inspect the proximal portion of the endovascular aortic graft, its proximal fixation stent, and its relationship to the renal arteries. On the other hand, Uchida et al. [15] described the history of the development of angioscopy, angioscopic systems and techniques by percutaneous angioscopy system which has been compared with imaging findings of IVUS.

In this study, we observed the state endoluminal SG in angioscopy which is a videoimage, and a possibility of angioscopy was indicated more than still image CT. We compared images from angioscopy with those taken from CT imaging, but a limit exists in picture of the image to show on the article and cannot show utility of the true intersection of the angioscope. Whilst coronary angioscopy provides a smaller observational field of vision, when compared to diagnostic radiography, the merits of being able to visually recognize actual circumstances is invaluable in a clinical context.

\section{Conclusion}

Potential future applications of this procedure include elucidation of the mechanism of large vessel disease, observation of the endoluminal surface of the SGs, clarification of endoleak, or resolution, among others.

\section{Funding Information}

The authors received no financial support for the research and/or authorship of this article.

\section{Consent}

Written informed consent was obtained from the patient for publication of this case report and accompanying images.

\section{Competing Interests}

The authors declare that they have no competing interests.

\section{References}

[1] Spears, J.R., Marais, J. and Serur, J. (1983) In Vivo Coronary Angioscopy. Journal of the American College of Cardi- 
ology, 5, 1311-1314. http://dx.doi.org/10.1016/S0735-1097(83)80145-4

[2] Litvack, F., Grundfest, W.S. and Lee, M.E. (1985) Angioscopic Visualization of Blood Vessel Interior in Animals and Humans. Clinical Cardiology, 8, 65-70. http://dx.doi.org/10.1002/clc.4960080202

[3] Mizuno, K., Miyamoto, A. and Satomura, K. (1991) Angioscopic Coronary Macromorphology in Patients with Acute Coronary Disorders. Lancet, 337, 809-812. http://dx.doi.org/10.1016/0140-6736(91)92514-3

[4] White, C.J., Ramee, S.R. and Mesa, J.E. (1991) Percutaneous Coronary Angioscopy in Patients with Restenosis after Coronary Angioplasty. Journal of the American College of Cardiology, 17, 46B-49B. http://dx.doi.org/10.1016/0735-1097(91)90938-6

[5] deFeyter, P.J., Ozaki, Y. and Baptista, J. (1995) Ischemia-Related Lesion Characteristics in Patients with Stable or Unstable Angina: A Study with Intracoronary Angioscopy and Ultrasound. Circulation, 92, 1408-1413. http://dx.doi.org/10.1161/01.CIR.92.6.1408

[6] Takano, M., Mizuno, K. and Yokoyama, S. (2003) Changes in Coronary Plaque Color and Morphology by Lipid-Lowering Therapy with Atorvastatin: Serial Evaluation by Coronary Angioscopy. Journal of the American College of Cardiology, 42, 680-686. http://dx.doi.org/10.1016/S0735-1097(03)00770-8

[7] Hill, B.B., Neville, R. and Hyde, G.L. (1995) Angioscopic Evaluation of an Endoluminal Aortic Graft: The First Clinical Experience. Journal of Endovascular Therapy, 2, 248-254. http://dx.doi.org/10.1583/1074-6218(1995)002<0248:AEOAEA>2.0.CO;2

[8] Komatsu, S., Ohara, T. and Takahashi, S. (2015) Early Detection of Vulnerable Atherosclerotic Plaque for Risk Reduction OF Acute Aortic Rupture and Thromboemboli and Atheroemboli Using Non-Obstructive Angioscopy. Circulation Journal, 79, 742-750. http://dx.doi.org/10.1253/circj.CJ-15-0126

[9] Canaud, L., Alric, P. and Desgranges, P. (2010) Factors Favoring Stent-Graft Collapse after Thoracic Endovascular Aorticrepair. The Journal of Thoracic and Cardiovascular Surgery, 139, 1153-1157. http://dx.doi.org/10.1016/j.jtcvs.2009.06.017

[10] Sze, D.Y., Mitchell, R.S. and Miller, D.C. (2009) Infolding and Collapse of Thoracic Endoprostheses: Manifestations and Treatment Options. The Journal of Thoracic and Cardiovascular Surgery, 138, 324-333. http://dx.doi.org/10.1016/j.jtcvs.2008.12.007

[11] Nanto, S., Ohara, T. and Mishima, M. (1991) Coronary Angioscopy: A Monorail Angioscope with Movable Guide Wire. American Journal of Cardiac Imaging, 5, 1-5.

[12] Mehta, M., Sternback, Y. and Taggert, J.B. (2010) Long-Term Outcomes of Secondary Procedures after Endovascular Aneurysm Repair. Journal of Vascular Surgery, 52, 1442-1448. http://dx.doi.org/10.1016/j.jvs.2010.06.110

[13] Avgerinos, E.D., Chaer, R.A. and Makaroun, M.S. (2014) Type II Endoleaks. Journal of Vascular Surgery, 60, 13861391. http://dx.doi.org/10.1016/j.jvs.2014.07.100

[14] Lawrence, D.D., Charnsagavej, C. and Wright, K.C. (1987) Percutaneous Endovascular Graft: Experimental Evaluation. Radiology, 163, 357-360. http://dx.doi.org/10.1148/radiology.163.2.2951767

[15] Uchida, Y. and Uchida, Y. (2012) Advances in Angioscopic Imaging of Vascular Disease. World Journal of Cardiovascular Surgery, 2, 114-131. http://dx.doi.org/10.4236/wjcs.2012.24021

\section{Submit or recommend next manuscript to SCIRP and we will provide best service for you:}

Accepting pre-submission inquiries through Email, Facebook, Linkedin, Twitter, etc

A wide selection of journals (inclusive of 9 subjects, more than 200 journals)

Providing a 24-hour high-quality service

User-friendly online submission system

Fair and swift peer-review system

Efficient typesetting and proofreading procedure

Display of the result of downloads and visits, as well as the number of cited articles

Maximum dissemination of your research work

Submit your manuscript at: http://papersubmission.scirp.org/ 